\title{
The damping width of giant dipole resonances of cold and hot nuclei: a macroscopic model
}

\author{
S. F. Mughabghab and A. A. Sonzogni \\ Brookhaven National Laboratory, Upton, NY 11973-5000.
}

\begin{abstract}
A phenomenological macroscopic model of the Giant Dipole Resonance (GDR) damping width of cold- and hot-nuclei with ground-state spherical and near-spherical shapes is developed. The model is based on a generalized Fermi Liquid model which takes into account the nuclear surface dynamics. The temperature dependence of the GDR damping width is accounted for in terms of surface- and volume-components. Parameter-free expressions for the damping width and the effective deformation are obtained. The model is validated with GDR measurements of the following nuclides, ${ }^{39,40} \mathrm{~K},{ }^{42} \mathrm{Ca},{ }^{45} \mathrm{Sc},{ }^{59,63} \mathrm{Cu},{ }^{109-120} \mathrm{Sn},{ }^{147} \mathrm{Eu},{ }^{194} \mathrm{Hg}$, and ${ }^{208} \mathrm{~Pb}$, and is compared with the predictions of other models.
\end{abstract}

Key words: macroscopic GDR model; temperature and spin dependence of GDR damping width; quadrupole deformation; in-medium nucleon-nucleon cross section

PACS:24.30.Cz, 21.60.Ev, 25.70.Gh, 25.70.Jj

\section{Introduction}

A long-standing problem of considerable interest and intense debate in nuclear physics is the theoretical description of giant dipole resonances dealing with the temperature- and spin-dependence of the damping width. Previously, three approaches were followed to describe the temperature dependence of the GDR damping width: the Landau model of adiabatic coupling of the GDR to thermal shape fluctuations (TSF) [1-2] ; the Fermi liquid model (FLM), treated in the framework of the linearized Landau-Vlasov kinetic equation [3-5]; and the phonon damping model, whereby correlated particle-hole states are coupled to more complicated configurations, such as incoherent ph, pp, and hh states [6]. None of these models can successfully account for the detailed shape and magnitude of the measured GDR damping widths in the temperature range from 0 to $4 \mathrm{MeV}$. As an example, a statistical analysis of gamma-ray spectra 
produced by inelastic scattering of alpha particles on ${ }^{120} \mathrm{Sn}$ demonstrated that neither the TSF model nor the collisional damping model can describe in detail the data but probably a combination of the two models can result in better agreement with the measurements [7]. The TSF model, which attributes the temperature-dependence of the damping width to surface effects, predicts a dependence of the form $T^{1 / 2}$, where $T$ is the nuclear temperature. In contrast, the Fermi liquid model, which accounts for the temperature-dependence in terms of quasi-particle collisions in the nuclear interior, predicts a quadratic temperature dependence. In addition, recent theoretical results [8], which reported a small change in the GDR width (about a 14\%) in the temperature range $0-4 \mathrm{MeV}$, have generated renewed debate as regards the magnitude of the width calculated in the framework of the TSF model. This result is at variance with previous theoretical calculations [1-2].

The purpose of this Letter is to address this challenging problem by following a different approach, based on a macroscopic generalized Landau Fermi Liquid Model (GLFM) for zero sound mode, which was quite successful in unifying the quantitative description of the E1 photon strength functions of spherical and deformed nuclei [9-10]. We note here that the possible transition from zero to first sound propagation for the hot dipole mode has been suggested recently [11-12].

First, we briefly present our macroscopic model and then apply it to measured ground-state GDR damping widths to determine one global parameter of the model, which is related to the in-medium nucleon-nucleon scattering cross section. This parameter would shed some light on the open question of this cross section. Second, we derive a simple expression for the GDR damping width and average quadrupole deformation of excited nuclear states in the framework of the coupling of the GDR to surface vibrations, as described by the dipole quadrupole interaction. Then we determine and discuss the

global parameters of the model and follow it with validation on the basis of extensive measurements of GDR widths and deduced quadrupole deformations of hot rotating nuclei, as obtained from intermediate-energy heavy-ion collision measurements. Finally, we give a summary and conclusion.

\section{Nuclear Model}

According to Landau's Fermi liquid model, the spreading width has two components: one is due to the decay of particle-hole states; the other results from the collisions of quasi particles in the nuclear interior. The former component is weakly temperature-dependent, while the latter follows a quadratic temperature-dependence. In previous studies [9-10], the FLM expression of the GDR damping width was generalized by taking into consideration the 
contribution of the surface component, as described by the coupling of the GDR to quadrupole surface vibrations [13-14]. At the outset, we make the following assumptions: the dipole-quadrupole interaction term for the excited state on which the GDR is built has the same form as that of the ground state; the GDR mode is adiabatically coupled to quadrupole shape fluctuations; quadrupole deformation depends on temperature, as well as angular momentum; the effect of motional narrowing on the GDR is not considered [15]. There are limitations to the adiabetic coupling assumption at temperatures larger than about $3 \mathrm{MeV}$. With this in mind, we then can write the following equation [9-10]:

$$
\Gamma(A, T, J)=C_{1}\left(E_{G D R}^{2}+4 \pi^{2} T^{2}\right)+C_{2} \beta(A, T, J) E_{G D R}
$$

where $\Gamma(A, T, J)$ is the GDR damping width of a hot rotating nucleus with a nuclear temperature $\mathrm{T}$, angular momentum $\mathrm{J}$, and nuclear mass $\mathrm{A} ; E_{G D R}$ is the centroid energy of the GDR; $\beta(A, T, J)$ is the average, effective nuclear quadrupole deformation. Here, we note that the measurements and theory [7,16-17] showed that there is little change of $E_{G D R}$ with temperature and angular momentum. The above relation then can be written in terms of the ground-state damping width, $\Gamma_{0}$, in the following form :

$$
\Gamma(A, T, J)=\Gamma_{0}(A)+4 \pi^{2} C_{1} T^{2}+C_{2} E_{G D R}\left[\beta(A, T, J)-\beta_{0}(A)\right],
$$

where

$$
\Gamma_{0}(A)=C_{1} E_{G D R}^{2}+C_{2} E_{G D R} \beta_{0}(A) .
$$

We stress that in these expressions, $C_{2}$ is set to the theoretical value, $2.35 \sqrt{\frac{5}{8 \pi}}=$ 1.05, where 2.35 is a conversion factor from standard deviation to full-width at half maximum for a Gaussian distribution. In addition, the global parameter $C_{1}$ can be computed from the theoretically calculated in-medium nuleonnucleon (NN) cross section. For details, refer for example to [3]. However, because of the large uncertainty in this theoretically calculated cross section [18-19], the $C_{1}$ parameter is determined phenomenologically from the groundstate damping widths, as described in the next section; the result of the analysis gives $C_{1}=0.0131 \pm 0.004 \mathrm{MeV}^{-1}$.

From the results of the liquid drop model $[1-2,20]$, in combination with our previous findings on the reduced magnitude of the TSF damping width [9-10] required to fit the data, we derive an expression for the effective quadrupole deformation term on the right-hand side of eq. 2. Following [21], we assumed that the two mechanisms which produce the spin-induced and thermallyinduced damping widths are independent. Then the dipole-quadrupole width, 
$2.35 \sqrt{\frac{5}{8 \pi}} E_{G D R}\left(\beta(A, J, T)-\beta_{0}(A)\right)$, is equated to the sum of the widths, added in quadrature, due to thermal shape fluctuation and angular momentum. At this point, we recall from [9-10] that the TSF width of [2] had to be changed by one third of of its value, or equivalently by one half of the liquid drop

model prediction of [20], 2.35E $E_{G D R} \sqrt{\frac{T}{V}}$, in order to describe the ${ }^{120} \mathrm{Sn}$ and ${ }^{208} \mathrm{~Pb}$ experimental data by surface and volume components [9-10],

Then it follows that

$$
\beta(A, T, J)-\beta_{0}(A)=\sqrt{\frac{8 \pi}{5}}\left(\frac{T}{4 V_{0}}+\frac{25}{32} \frac{y^{2}}{(1-x)^{2}}\right)^{1 / 2},
$$

where:

$$
\begin{aligned}
& V_{0}=0.80 a_{s} A^{2 / 3}(1-x), \\
& x=0.019 \frac{Z^{2}}{A}\left(1-1.7826 \frac{N-Z}{A}\right)^{-1}, \\
& y=2.1 A^{-7 / 3} J^{2} \frac{I_{\text {rigid }}}{I_{\text {eff }}},
\end{aligned}
$$

$a_{s}$ is the surface energy, $a_{s}=17.94 \mathrm{MeV}[22], I_{\text {eff }}$ is the effective moment of inertia, and $I_{\text {rigid }}$ is the moment of inertia of a rigid rotor. The spin- and deformation-dependence of $I_{\text {eff }}$ was taken into account by examining the super deformed rotational bands and by following the prescription of [14].

In the absence of measurements for ground state GDR widths, such as ${ }^{45} \mathrm{Sc}$, ${ }^{147} \mathrm{Eu}$, and ${ }^{194} \mathrm{Hg}$, eq. 3 was applied in this evaluation. We emphasize that eq. 2 - eq. 3 are parameter free, except for the global parameter, $C_{1}$, which is derived from measured ground-state widths.

\section{In-medium nucleon-nucleon scattering cross section}

Although two different theoretical approaches were able to reproduce reasonably well the free-space nucleon-nucleon scattering cross section for laboratory energies 0 -300 MeV, a large disagreement resulted in the predicted in-medium cross section; for details see [18-19]. In one model [18], the in-medium NN cross 
section is appreciably reduced from its value in free-space throughout this energy region. In the other [19], the predicted in-medium NN cross section shows a resonance behavior at laboratory energy of about $90 \mathrm{MeV}$ for nuclear densities of half the saturation value. Specifically, the cross section is suppressed below about $50 \mathrm{MeV}$ and is enhanced relative to the free one in the energy region $50 \leq \mathrm{E}_{l a b} \leq 130$. Because of this large disagreement in the predictions [18-19] of the in-medium NN cross section, the global parameter $C_{1}$ is determined here from the extensive measured data of ground-state GDR widths $(\mathrm{T}=0)$ of spherical- and near-spherical nuclei of nuclear masses from 40 to 209 [23] by a non-linear least-squares fitting procedure. The result of the analysis on the basis of eq. 3 yields $C_{1}=0.0131 \pm 0.004 \mathrm{MeV}^{-1}$; the uncertainty of the constant corresponds to $95 \%$ confidence interval. This value is in excellent agreement with a calculated value of $0.0135 \mathrm{MeV}^{-1}$, derived on the basis of a coss section of $50 \mathrm{mb}$ in the CM system [3].

From our result, it follows that a cross section of $49 \pm 2 \mathrm{mb}$ is obtained for the in-medium NN cross section at the Fermi enegy, which is not in agreement with the theoretical values of [18-19]. The error reflects the uncertainty in $C_{1}$ and does not include the theoretical uncertainty due to the approximations, which is difficult to assess. The present result shows that the in-medium NN cross section is neither suppressed nor enhanced relative to the free-space one. In addition, we would like to remark that the $C_{1}$ value can also be easily obtained from the measured temperature dependence of the GDR widths for low angular momenta with the help of eq. 2 .

\section{Validation of the Model}

At the start, the validity of the temperature dependence of the quadrupole deformation for low angular momenta, according to eq. 4, is tested by comparing its estimates with the TSF predictions for ${ }^{120} \mathrm{Sn}$ [8]. The results are displayed in fig.1. As shown, very good agreement between both calculations is obtained.

Next, our model is tested by comparing its predictions of $\Gamma(A, T, J)$ and $\beta(A, T, J)$ with experimental values for a wide range of hot rotating nuclei. Experimental studies have been carried out for the following cases: ${ }^{39,40} \mathrm{~K},{ }^{42} \mathrm{Ca}$, ${ }^{45} \mathrm{Sc},{ }^{59,63} \mathrm{Cu},{ }^{109-120} \mathrm{Sn},{ }^{147} \mathrm{Eu},{ }^{194} \mathrm{Hg},{ }^{208} \mathrm{~Pb}$. The outcome of the comparison is a remarkable agreement between our model's predictions and the experimental data. Due to space limitations, however, we will restrict our discussion to five representative cases, ${ }^{45} \mathrm{Sc},{ }^{109} \mathrm{Sn},{ }^{118} \mathrm{Sn},{ }^{147} \mathrm{Eu}$, and ${ }^{194} \mathrm{Hg}$. For these nuclei, equilibrium deformation is sustained up to an angular momentum of $60 \hbar$. 


\section{$4.1{ }^{45} \mathrm{Sc}$}

The experimental values [24] are shown in the left panels of fig. 2. The quadrupole deformations were deduced in [24] from the energy splitting of the GDR peak. The solid and dashed lines are our model predictions for two temperatures, 1.7 $\mathrm{MeV}$ and $2.3 \mathrm{MeV}$, respectively corresponding to the temperature range of the measurements [24]. It is of interest to note that the GDR widths for $\langle J\rangle=13 \hbar, 18.5 \hbar$ and $\langle J\rangle=21.4 \hbar, 23.5 \hbar$ line up with the curves associated with temperatures of $1.7 \mathrm{MeV}$ and $2.3 \mathrm{MeV}$ respectively, in agreement with measurements [24] (left-top panel). In addition, the predicted deformations in the spin range from $13 \hbar$ to $23.5 \hbar$ (left-bottom panel) are in agreement with the measurenents [24].

\section{2 ${ }^{109} \mathbf{S n}$}

Measurements were performed at angular momenta ranging from $\langle J\rangle=10 \hbar$ to $54 \hbar$, and average temperatures from $1.4 \mathrm{MeV}$ to $1.8 \mathrm{MeV}$ [25-26]. The topright panel of fig. 2 displays the experimental widths along with our model predictions for $T=1.4 \mathrm{MeV}$ (solid line) and $T=1.8 \mathrm{MeV}$ (dashed line). The bottom-right panel shows our predictions for the deformation as a function of angular momentum at two temperatures, $1.4 \mathrm{MeV}$ and $1.8 \mathrm{MeV}$. Since only one Lorentzian shape fit was made to the GDR, the experimental deformation parameters for this nucleus were not determined.

\section{$4.3 \quad{ }^{147} \mathbf{E u}$}

Measurements were performed at an average temperature around $1.3 \mathrm{MeV}$ and angular momenta in the range $\langle J\rangle=37 \hbar$ to $55 \hbar$ [27]. The $\beta$ values were deduced from the energy splitting of the two Lorentzian fits [27]. The widths (deformations) are displayed in the left-top (left-bottom) panel of fig. 3 The solid lines are our model calculations at $T=1.3 \mathrm{MeV}$. The reported TSF predictions, calculated at two temperatures of $1.2 \mathrm{MeV}$ and $1.4 \mathrm{MeV}$, are represented by dot and dot-dash lines respectively [27].

\section{$4.4 \quad{ }^{194} \mathbf{H g}$}

This nucleus exemplifies the decreasing influence of the moment of inertia on the GDR width with increasing nuclear mass. As shown on the top-right panel

of fig. 3, the measured GDR widths at $\langle J\rangle=24 \hbar, 27 \hbar, 36 \hbar$ and average $T$ of 
1.3 MeV exhibit a constant value of $6.2 \pm 0.5 \mathrm{MeV}$ [28]. Our model reproduces the observed constancy of the width in this $J$ range and gives an estimate of 6.6 $\mathrm{MeV}$ for the GDR width in this spin range.

\section{$4.5{ }^{118} \mathrm{Sn}$}

To illustrate the dependence of the GDR width on temperature and angular momentum, we summarized in fig. 4 the available experimental results for ${ }^{118} \mathrm{Sn}$ and nearby tin nuclei [29-35]. This problem was recently investigated in [29]. The dashed line, which is reproduced from [29], represents the TSF predictions. This is to be compared with our estimate, described by the solid line. In carrying out these calculations, we took into consideration the dependence of the angular momentum on temperature as reported in [29].

\section{$5 \quad$ Summary and conclusion}

In the present detailed study, we demonstrated that our new approach, which is based on a generalized Landau Fermi liquid model, is successful in well describing the dependence of the GDR damping width on the nuclear temperature up to $3 \mathrm{MeV}$ and angular momentum up to $60 \hbar$ for a wide range of hot rotating nuclei. Two very rewarding features of the model are its simplicity and its accuracy. We have also shown that the influence of the angular momentum on the GDR width comes into play at particular values depending on the nuclear mass. For A around 45, 110, and 180, these angular momenta are $J=10 \hbar, 30 \hbar$ and $40 \hbar$ respectively. In addition, we derived a simple parameter-free expression for the quadrupole deformation of excited nuclear states, which was tested and validated with ${ }^{45} \mathrm{Sc}$ and ${ }^{147} \mathrm{Eu}$ measured data. The most significant results of this study are: a) the GDR width of hot rotating nuclei can be well explained in terms of two mechanisms, the collisional damping model and the thermal shape fluctuations model; b) the GDR width contains fundamental nuclear information, such as the effective nuclear deformation and the in-medium NN cross section which can be readily deduced from our simple model; c) this model can be applied to other finite Fermi systems and other vibrational modes, such as the giant quadrupole and octupole resonances, which presently are under investigation. An important by-product of the present investigation is the determination of the in-medium nucleonnucleon cross section at the Fermi energy, which shows that this cross section is neither suppressed or enhaned from the one in free-space. 


\section{Acknowledgements}

The authors gratefully acknowledge fruitful discussions with W. E. Ormand, and critical reading of the manuscript by P. Oblozinsky. This research was

carried out under the auspices of the US Department of Energy under Prime Contract No. DE-AC02-98CH10886.

\section{References}

[1] W.E. Ormand, P.F. Bortignon, and R.A. Broglia, Phys. Rev Lett. 77 (1996) 607.

[2] D. Kusnezov, Y. Alhassid, and K.A. Snover, Phys. Rev Lett. 81 (1998) 542.

[3] M. Di Toro, V.M. Kolomietz and A.B. Larionov, Phy. Rev. C 59 (1999) 3099.

[4] U. Fuhrmann, K. Morawetz, and R. Walk, Phy. Rev. C 58 (1998) 1473.

[5] O. Yilmaz et al., Phys. Lett. B 472 (2000) 258.

[6] N. Dinh Dang, K. Tanabe, and A. Arima, Nucl. Phys. A645 (1999) 536.

[7] G. Gervais, M. Thoennessen, and W.E. Ormand, Phys. Rev. C 58 (1998) R1377.

[8] A. Ansari, N. Dinh Dang, and A. Arima, Phys. Rev. C 62 (2000) 011302R.

[9] S.F. Mughabghab and C.L. Dunford, Phys. Lett. B 487 (2000) 155.

[10] S.F. Mughabghab and C.L. Dunford, in Proceedings of the XIII Conference on Nuclear Physics, Neutron Physics and Nuclear Energy, Bulgarian Nucl. Soc. 5, 99 (2000).

[11] A. Larionov et al. Nucl. Phys. 648A (1999) 157.

[12] V. Baran et al. Nucl. Phys.A 599 (1996) 29c.

[13] J. Le Tourneux, Mat. Fys. Medd. Dan. Vid. Selsk. 34, No 11 (1965).

[14] A. Bohr and B.R. Mottelson, Nuclear Structure 2, Benjamin, New York (1969).

[15] W. E. Ormand et al. Phys. Rev. Lett. 64 (1990) 2254.

[16] K. Snover, Ann. Rev. Nucl. Part. Sci. 36 (1986) 545.

[17] J.J. Gaardhoje, Ann. Rev. Nucl. Part. Sci. 42 (1992) 483.

[18] G. Q. Li and R. Machleid, Phys. Rev. C 49 (1994) 566.

[19] T. Alm, G. Ropke, and M. Schmidt, Phys. Rev. C 50 (1994) 31.

[20] S.S. Dietrich and B.L. Berman, At. Data and Nucl. Data Tables 38 (1988) 199.

[21] R.A. Broglia, W.E. Ormand and M. Borromeo, Nucl. Phys. A482 (1988) 141c. 
[22] R. A. Broglia, P. F. Bortignon, and A. Bracco, Prog. Part. Nucl. Phys. 28 (1992) 517.

[23] J.R. Nix, Ann. Rev. Nucl. Sci. 22 (1972) 65.

[24] M. Kicinska-Habior et al., Phys. Lett. B 308, (1993) 225.

[25] A. Bracco et al., Phys. Rev. Lett. 74, (1995) 3748.

[26] M. Mattiuzzi et al., Nucl. Phys. A612, (1997) 262.

[27] M. Kmiecik et al., Nucl. Phys. A674, (2000) 29.

[28] F. Camera et al., Phys. Rev. C 60, (1999) 014306.

[29] M.P. Kelly et al., Phys. Rev. Lett. 82, (1999) 3404.

[30] A. Bracco et al., Phys. Rev. Lett. 62, (1989) 2080.

[31] G. Enders et al., Phys. Rev. Lett. 69, (1992) 249.

[32] J.J. Gaardhoje et al., Phys. Rev. Lett. 53, (1984) 148.

[33] J.J. Gaardhoje et al., Phys. Rev. Lett. 56, (1986) 1783.

[34] D.R. Chakrabarty et al., Phys. Rev. C. 36, (1987) 1886.

[35] K.A. Snover, in Future Directions in Nuclear Physics with $4 \pi$ Gamma Detection Systems of the New Generation, edited by J. Dudek and B. Hass, AIP Conf. Proc. No 259 (AIP, New York, 1992), p.229. 


\section{Figure Captions}

Fig. 1

Quadrupole deformation $(\beta)$ as a function of nuclear temperature $(\mathrm{T})$ for

${ }^{120} \mathrm{Sn}$. The solid and dash lines represent our model predictions and those of [8] respectively. For details, see the text.

Fig. 2

GDR widths $(\Gamma)$ and quadrupole deformations $(\beta)$ as a function of angular momentum for ${ }^{45} \mathrm{Sc}$ (left panels) and ${ }^{109} \mathrm{Sn}$ (right panels). The solid and dash lines represent our model predictions at two temperatures. The data points with uncertainties are from [24] for ${ }^{45} \mathrm{Sc}$ and from $[25-26]$ for ${ }^{109} \mathrm{Sn}$. For details, see the text.

Fig. 3

GDR widths $(\Gamma)$ and quadrupole deformations $(\beta)$ as a function of angular momentum for ${ }^{147} \mathrm{Eu}$ (left panels) and ${ }^{194} \mathrm{Hg}$ (right panels). The data points with uncertainties are obtained from [27] and [28] for ${ }^{147} \mathrm{Eu}$ and ${ }^{194} \mathrm{Hg}$ respectively. The solid lines are our model predictions at a nuclear temperature of $1.3 \mathrm{MeV}$. The dot and dot-dash lines are the thermal shape fluctuations predictions (TSF) [27] at temperatures of $1.2 \mathrm{MeV}$ and $1.4 \mathrm{MeV}$ respectively.

Fig. 4

GDR width $(\Gamma)$ as a function of temperature for ${ }^{118} \mathrm{Sn}$ and nearby tin nuclei. The measurements are obtained from [29] and references therein. The solid line is our model prediction and is compared with the TSF calculations of [29] (dash line). 


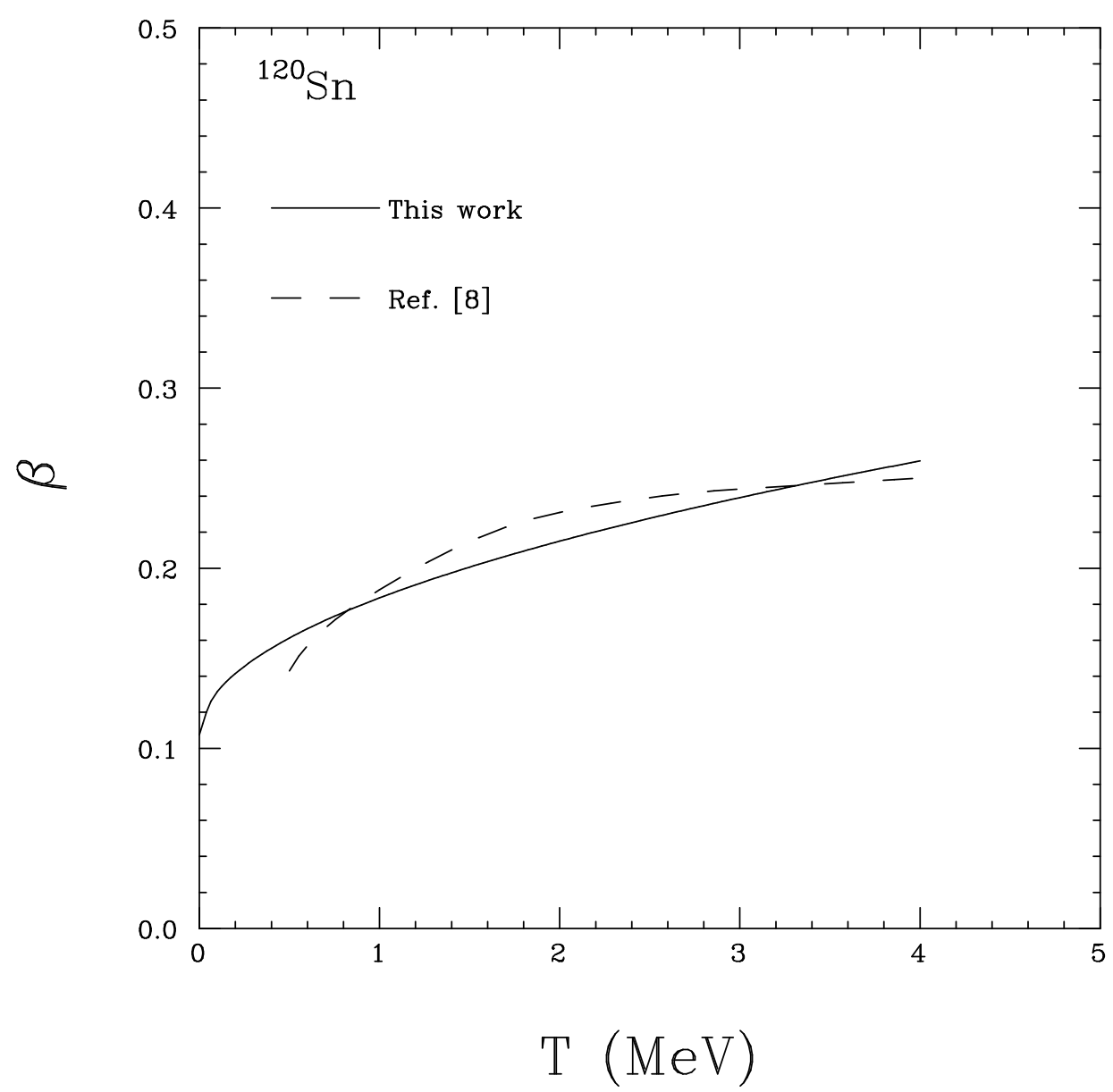




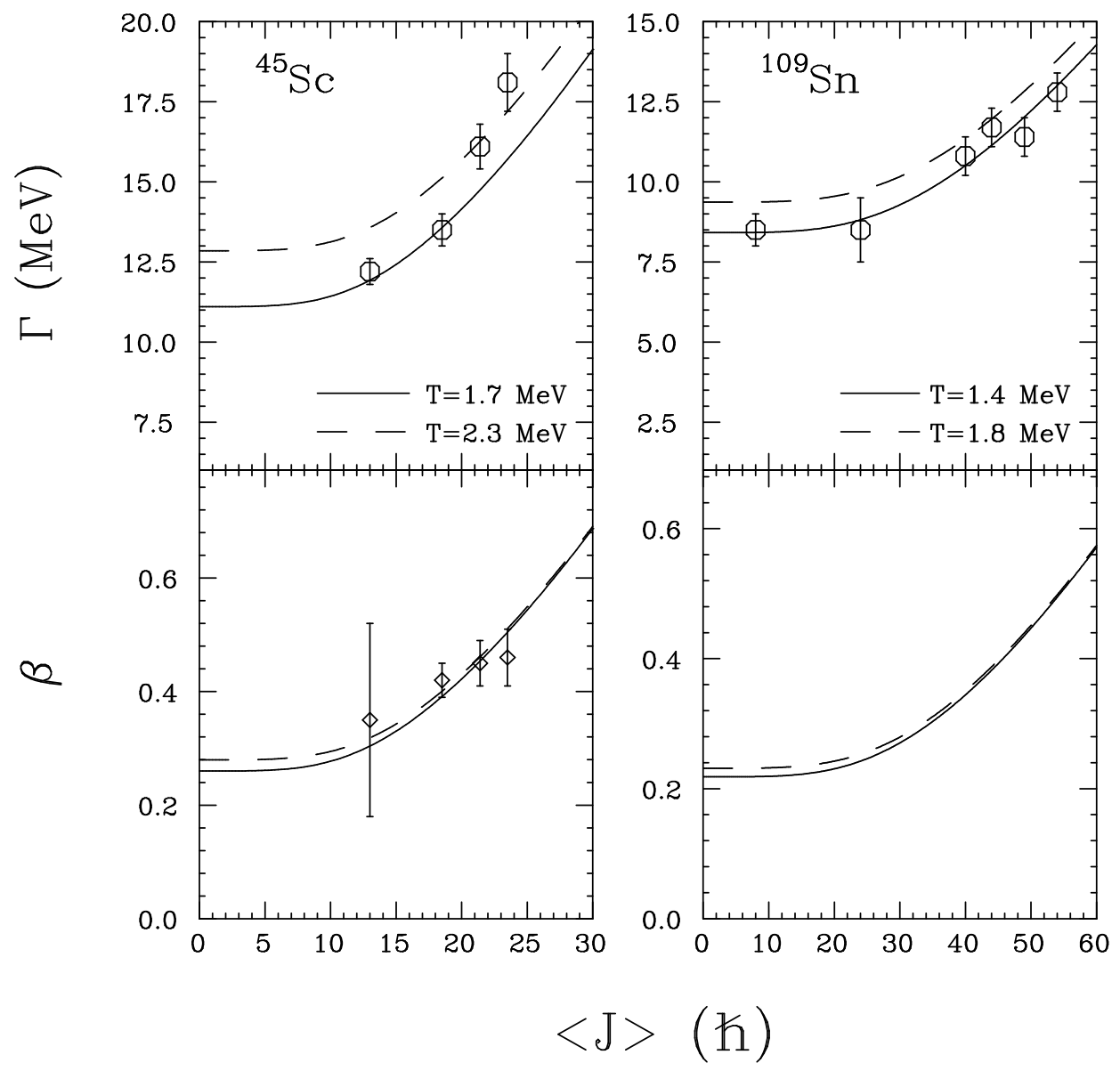




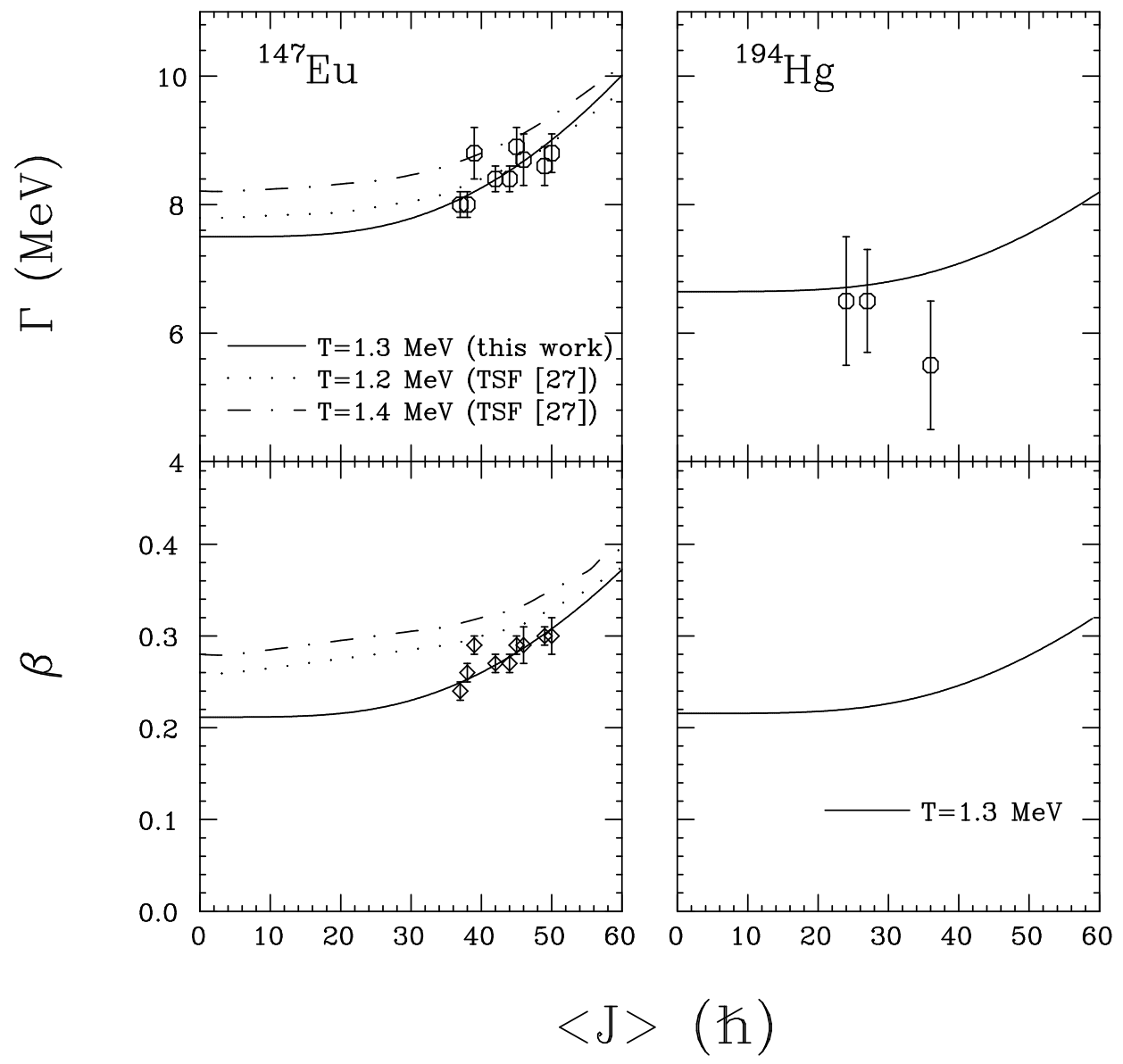




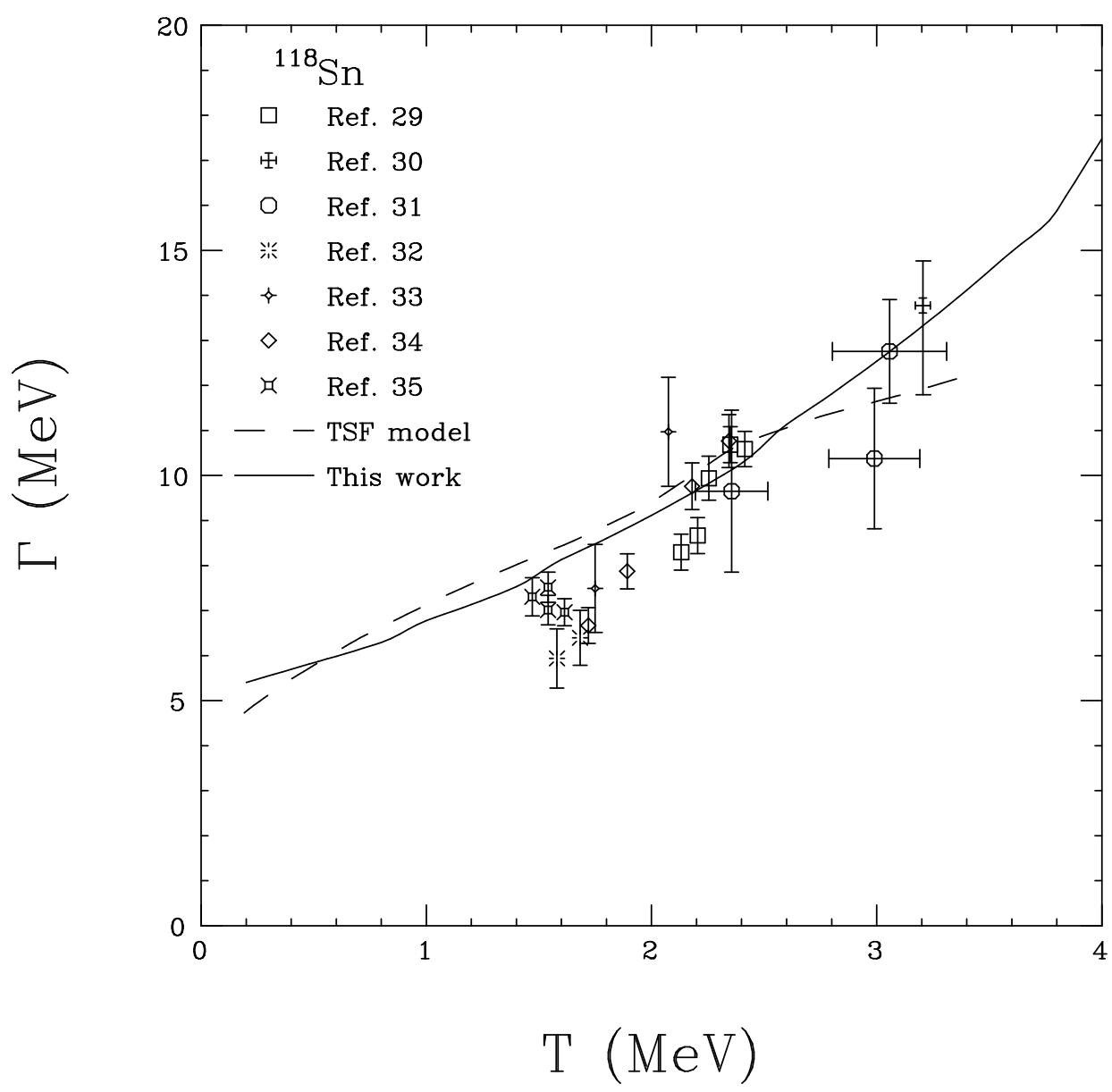

